TAIWANESE JOURNAL OF MATHEMATICS

Vol. 11, No. 3, pp. 637-643, August 2007

This paper is available online at http://www.math.nthu.edu.tw/tjm/

\title{
A THIRD ORDER EQUATION ARISING IN THE FALLING FILM
}

\author{
Yung-Jen Lin Guo
}

\begin{abstract}
We study a third-order differential equation arising in the study of falling film on a coated vertical fibre. We first provide some properties of solutions to this third-order equation. Then we prove some nonexistence results of global solution to this equation under certain boundary condition at infinity.
\end{abstract}

\section{INTRODUCTION}

In this paper, we shall study the following third order ordinary differential equation

$$
\phi^{\prime \prime \prime}(y)+\phi^{\prime}(y)+g(\phi(y))=0, \quad \phi(y)>0, \quad y \in \mathbb{R},
$$

where $g$ is a nonlinear function satisfying the following condition

$$
g^{\prime}>0 \text { in }(0, \infty), \quad g\left(0^{+}\right)=-\infty, \quad g(1)=0, \quad g(+\infty) \in(0, \infty) .
$$

The equation (1.1) arises in the study of a falling film on a coated vertical fibre. If we denote by $h=h(x, t)$ the thickness of the falling film on the coated vertical fibre with the positive $x$-axis pointing downward, then $h$ satisfies the following fourth order parabolic partial differential equation:

$$
h_{t}+\left[\delta h^{3}\left(h_{x x x}+h_{x}\right)+\frac{2}{3} h^{3}\right]_{x}=0, x \in \mathbb{R}, t>0,
$$

where $\delta$ is a positive constant which measures the ratio of curvature-driven flow of the Rayleigh instability to the gravity-driven mean flow. For more detailed physical

Received November 28, 2006

Communicated by J. C. Yao.

2000 Mathematics Subject Classification: 34A12, 35K30.

Key words and phrases: Third-order differential equation, Falling film, Initial value problem, Global solutions.

This work was partially supported by the National Science Council of the Republic of China under the grant NSC 94-2115-M-003-009. 
background and the derivation of (1.3), we refer the reader to $[3,2,5]$ and the references cited therein.

In the study of the above falling film problem, it is important and interesting to find traveling wave solutions of (1.3) in the form $h(x, t):=\phi(x-c t)$ with wave speed $c \geq 0$. If we set $y:=x-c t$, then $h$ satisfies (1.3) if and only if $\phi$ satisfies

$$
-c \phi^{\prime}+\left[\delta \phi^{3}\left(\phi^{\prime \prime \prime}+\phi^{\prime}\right)+\frac{2}{3} \phi^{3}\right]^{\prime}=0 \quad \text { in } \mathbb{R} .
$$

Assuming $h(x, t) \rightarrow 1$ as $x \rightarrow \pm \infty$, then, by an integration, $\phi$ satisfies (1.1) with $g$ given by

$$
g(\phi)=\frac{1}{\delta}\left[\frac{2}{3}-c \phi^{-2}-\left(\frac{2}{3}-c\right) \phi^{-3}\right] .
$$

We note that $g(1)=0$ and $g(+\infty)=2 /(3 \delta)$ for all $c$. Note that $g$ satisfies (1.2) when $c \in[0,2 / 3]$.

The study of third-order differential equations has attracted a lot of attentions for the past years. These equations arise in many different applications, such as the free convection problem in boundary layer theory [4] and the problem of coating and draining flows $[1,6,7]$.

In this paper, we shall prove some nonexistence results for global solutions of (1.1) with the boundary condition $\phi( \pm \infty)=1$, under the condition (1.2). There is a simple application of this result to the falling film problem. That is, a traveling wave solution of (1.3) exists only if the wave speed is bigger than $2 / 3$.

This paper is organized as follows. In the next section, we shall give some properties of solutions of (1.1). Then we prove some nonexistence results of global solution of (1.1).

\section{Preliminary}

In this section, we provide some properties of solutions of (1.1).

Lemma 2.1. Suppose that $\phi$ is a solution of (1.1) with $\phi\left(y_{0}\right)=1$ and $\phi^{\prime}>0$ in $\left(y_{0}, \infty\right)$ for some $y_{0} \in[-\infty, \infty)$. Then $\phi(y) \rightarrow \infty$ as $y \rightarrow \infty$.

Proof. Let $l:=\lim _{y \rightarrow \infty} \phi(y)$. Then $l>1$. Suppose for contradiction that $l<\infty$. By an integration of (1.1), we obtain

$$
\phi^{\prime \prime}(y)-\phi^{\prime \prime}(z)+\phi(y)-\phi(z)+\int_{z}^{y} g(\phi(s)) d s=0 .
$$

Taking a fixed $z>y_{0}$ and letting $y \rightarrow \infty$ in (2.1), we see that $\phi^{\prime \prime}(y) \rightarrow-\infty$ as $y \rightarrow \infty$, contradicting the fact that $\phi^{\prime}>0$ in $\mathbb{R}$. Hence $l=\infty$ and the lemma follows. 
Lemma 2.2. There is no solution of (1.1) with $\phi\left(y_{0}\right)=1$ and $\phi^{\prime}>0$ in $\left(y_{0}, \infty\right)$ for some $y_{0} \in[-\infty, \infty)$.

Proof. Suppose that there is a solution $\phi$ with $\phi\left(y_{0}\right)=1$ and $\phi^{\prime}>0$ in $\left(y_{0}, \infty\right)$ for some $y_{0} \in[-\infty, \infty)$. Then by Lemma 2.2, we have $\phi(y) \rightarrow \infty$ as $y \rightarrow \infty$. Since $\phi^{\prime}>0$ in $\left(y_{0}, \infty\right)$, we can invert $\phi=\phi(y)$ as $y=y(\phi)$. We introduce the function

$$
u(\phi)=\left[\phi^{\prime}(y)\right]^{2}, y=y(\phi) .
$$

Then $u=u(\phi)>0$ for $\phi \in(1, \infty)$ and $u$ satisfies the second order ODE:

$$
u^{\prime \prime}=-2-g(\phi) \frac{2}{\sqrt{u}}, \phi \in(1, \infty) .
$$

Since $g>0$ in $(1, \infty)$, we have $u^{\prime \prime}<-2$ in $(1, \infty)$. This implies that $u$ vanishes at some finite $\phi_{0}$, a contradiction. Hence the lemma is proved.

As a corollary of Lemma 2.2, we obtain the following property of solution of (1.1).

Corollary 2.3. Let $\phi$ be a solution of (1.1) with $\phi\left(y_{0}\right)=1$ and $\phi^{\prime}>0$ in $\left(y_{0}, y_{0}+\delta\right)$ for some $y_{0} \in[-\infty, \infty)$ and $\delta>0$. Then $\phi^{\prime}$ vanishes at some finite point $y>y_{0}$.

Next, we study the asymptotic behavior of global solution of (1.1) as $x \rightarrow \pm \infty$.

Lemma 2.4. Suppose that $l:=\lim _{x \rightarrow \infty} \phi(x)$ exists. Then $l=1$.

Proof. Suppose that $l \neq 1$. Then we may choose $z$ sufficiently large such that $g(\phi(y))$ has a fixed sign for all $y \geq z$. Thus the limit

$$
I:=\int_{z}^{\infty} g(\phi(s)) d s
$$

exists and is either $\infty$ or $-\infty$. It follows from (2.1) that the $\operatorname{limit} L:=\lim _{y \rightarrow \infty} \phi^{\prime \prime}(y)$ also exists and either $L=\infty$, if $I=-\infty$, or $L=-\infty$, if $I=\infty$.

Now, if $L=\infty$, then $\phi^{\prime}(y) \rightarrow \infty$ as $y \rightarrow \infty$ and so $l=\infty$. This implies that $I=\infty$, a contradiction. If $L=-\infty$, then $\phi^{\prime}(y) \rightarrow-\infty$ as $y \rightarrow \infty$. This implies that $\phi$ vanishes at some finite $y$, contradicting to the fact that $\phi>0$ on $\mathbb{R}$. Therefore, $l=1$ and the lemma follows.

Similarly, we can prove that

Lemma 2.5. If the limit $l:=\lim _{x \rightarrow-\infty} \phi(x)$ exists and $l<\infty$, then $l=1$. 


\section{Main Results}

In this section, we shall derive the nonexistence of certain global solutions of (1.1).

Theorem 3.1. There is no global solution $\phi$ of (1.1) with $\phi<1$ in $\mathbb{R}$.

Proof. Suppose that there is a global solution $\phi$ of (1.1) with $\phi<1$ in $\mathbb{R}$. Let

$$
l^{+}:=\limsup _{y \rightarrow \infty} \phi(y), l^{-}:=\limsup _{y \rightarrow-\infty} \phi(y) .
$$

We claim that $l^{+}=l^{-}=1$.

If $l^{+}<1$, then there is a $z>0$ such that $\phi(y) \leq\left(1+l^{+}\right) / 2$ for all $y \geq z$. Hence

$$
\int_{z}^{\infty} g(\phi(s)) d s=-\infty
$$

and so $\phi^{\prime \prime}(y) \rightarrow \infty$ as $y \rightarrow \infty$ by (2.1). Then $\phi^{\prime}(y) \rightarrow \infty$ as $y \rightarrow \infty$ and $\phi(y)>1$ for all $y$ sufficiently large, a contradiction to the above inequality. Therefore, we have $l^{+}=1$.

Similarly, $l^{-}=1$. Note that the same argument leads that

$$
\int_{z}^{\infty} g(\phi(s)) d s>-\infty, \int_{-\infty}^{z} g(\phi(s)) d s>-\infty
$$

for any $z \in \mathbb{R}$. Hence it follows from (2.1) that $\phi^{\prime \prime}$ is bounded in $\mathbb{R}$.

Next, we claim that $\phi^{\prime}(y)>0$ for all $y$ sufficiently large, $\phi^{\prime}(y)<0$ for all $-y$ sufficiently large, and so $\lim _{y \rightarrow \pm \infty} \phi(y)=1$.

Suppose on the contrary that there is a sequence $\left\{y_{n}\right\}$ such that $y_{n} \rightarrow \infty$ and $\phi^{\prime}\left(y_{n}\right)=0$ for all $n \geq 1$. We may assume that $\phi^{\prime \prime}\left(y_{2 k}\right) \geq 0$ and $\phi^{\prime \prime}\left(y_{2 k+1}\right) \leq 0$ for all $k \geq 1$ and $\phi\left(y_{2 k+1}\right) \rightarrow 1$ as $k \rightarrow \infty$. Introduce

$$
\Phi_{1}(y):=\Phi_{1}(y ; \phi)=\phi^{\prime \prime}(y)[\phi(y)-1]-\frac{1}{2}\left[\phi^{\prime}(y)\right]^{2}+\frac{1}{2}[\phi(y)-1]^{2} .
$$

Note that

$$
\Phi_{1}^{\prime}(y)=-g(\phi(y))[\phi(y)-1]
$$

Hence $\Phi_{1}$ is monotone decreasing in $y$. Set

$$
L^{+}:=\lim _{y \rightarrow \infty} \Phi_{1}(y)
$$

Note that $\phi^{\prime \prime}$ is bounded. Since $\Phi_{1}\left(y_{2 k+1}\right) \geq\left[\phi\left(y_{2 k+1}\right)-1\right]^{2} / 2>0$ for all $k$, we have $L^{+} \in[0, \infty)$. Since $\phi\left(y_{2 k+1}\right) \rightarrow 1$ as $k \rightarrow \infty$ and $\phi^{\prime \prime}$ is bounded, we 
have $\Phi_{1}\left(y_{2 k+1}\right) \rightarrow 0$ as $k \rightarrow \infty$. Hence $L^{+}=0$. Similarly, by taking a sequence $y_{n} \rightarrow-\infty$ such that $\phi\left(y_{n}\right) \rightarrow 1$ as $n \rightarrow \infty$ and using the fact that $\phi^{\prime \prime}$ is bounded, we get that

$$
L^{-}:=\lim _{y \rightarrow-\infty} \Phi_{1}(y)=\lim _{n \rightarrow \infty} \Phi_{1}\left(y_{n}\right)=\lim _{n \rightarrow \infty}\left\{-\frac{1}{2}\left[\phi^{\prime}\left(y_{n}\right)\right]^{2}\right\} \leq 0 .
$$

This contradicts the decreasing property of $\Phi_{1}$. Therefore, $\phi$ can only have finitely many critical points in $(0, \infty)$. Then $\lim _{y \rightarrow \infty} \phi(y)=1$ and $\phi^{\prime}(y)>0$ for all $y$ sufficiently large.

Similarly, $\phi^{\prime}(y)<0$ for all $-y$ sufficiently large and $\lim _{y \rightarrow-\infty} \phi(y)=1$.

Now, we can choose a sequence $\left\{y_{n}\right\}$ such that $y_{n} \rightarrow \pm \infty$ and $\phi^{\prime}\left(y_{n}\right) \rightarrow 0$ as $n \rightarrow \pm \infty$. Since $\phi^{\prime \prime}$ is bounded, it follows that $\Phi_{1}\left(y_{n}\right) \rightarrow 0$ as $n \rightarrow \pm \infty$. This again contradicts the decreasing property of $\Phi_{1}$. This completes the proof of the theorem.

Theorem 3.2. There is no global solution $\phi$ of (1.1) with $\phi>1$ in $\mathbb{R}$ and $\phi$ is bounded in $(-\infty, 0)$.

Proof. The proof is similar to the one for Theorem 3.1 and we omit it.

As a consequence of Theorems 3.1 and 3.2, we have

Corollary 3.3. Any global solution of (1.1) which is bounded in $(-\infty, 0)$ must take the value 1 .

Notice that any global solution of (1.1) with $\phi(-\infty)=1$ is bounded in $(-\infty, 0)$.

Lemma 3.4. Let $\phi$ be a nontrivial global solution of (1.1). Then any point $y_{0}$ with $\phi\left(y_{0}\right)=1$ cannot be an accumulation point of the set $\Sigma:=\{y \mid \phi(y)=1\}$.

Proof. Otherwise, there is a distinct sequence $\left\{y_{n}\right\} \subset \Sigma$ such that $y_{n} \rightarrow y_{0}$ as $n \rightarrow \infty$. Then $\phi^{\prime}\left(y_{0}\right)=0$. Hence $\Phi_{1}\left(y_{0}\right)=0$. Since $\Phi_{1}\left(y_{n}\right) \leq 0$ for all $n$, it follows from the decreasing property of $\Phi_{1}$ that $y_{n}>y_{0}$ for all $n$.

On the other hand, since $\phi$ is nontrivial, $\phi^{\prime \prime}\left(y_{0}\right) \neq 0$. If $\phi^{\prime \prime}\left(y_{0}\right)>0$, then $\phi(y)>1$ for all $y>y_{0}$ with $y-y_{0}$ small, a contradiction. If $\phi^{\prime \prime}\left(y_{0}\right)<0$, then $\phi(y)<1$ for all $y>y_{0}$ with $y-y_{0}$ small, again a contradiction. The lemma follows.

Lemma 3.5. Let $\phi$ be a global solution of (1.1) which is bounded in $(-\infty, 0)$. Then there is a sequence $y_{n}$ such that $y_{n} \rightarrow \infty$ and $\phi\left(y_{n}\right)=1$ for all $n$.

Proof. Set $\bar{y}:=\sup \{y \mid \phi(y)=1\}$. We claim that $\bar{y}=\infty$.

On the contrary, we assume that $\bar{y}<\infty$. Then $\phi(\bar{y})=1$. 
Case 1. $\phi(y)>1$ for all $y>\bar{y}$. Then $\phi^{\prime}(\bar{y}) \geq 0$ and $\phi^{\prime \prime}(\bar{y})>0$ if $\phi^{\prime}(\bar{y})=0$. By Corollary 2.3, there is the smallest $y_{0}>\bar{y}$ such that $\phi^{\prime}\left(y_{0}\right)=0, \phi^{\prime \prime}\left(y_{0}\right) \leq 0$, and $\phi^{\prime}(y)<0$ for all $y>y_{0}$ with $y-y_{0}$ small. Recall that $\Phi_{1}(\bar{y}) \leq 0$. Then $\phi^{\prime}(y)<0$ for all $y>y_{0}$. Otherwise, we have $\phi^{\prime \prime}\left(y_{1}\right) \geq 0$ for the smallest critical point $y_{1}>y_{0}$. Note that $\phi\left(y_{1}\right)>1$. This implies that $\Phi_{1}\left(y_{1}\right)>0$, a contradiction. Therefore, $\phi^{\prime}(y)<0$ for all $y>y_{0}$. It follows from Lemma 2.4 that $\phi(y) \rightarrow 1$ as $y \rightarrow \infty$.

By (2.1), we have $0<\int_{\bar{y}}^{\infty} g(\phi(s)) d s<\infty$ and $\phi^{\prime \prime}$ is bounded in $[\bar{y}, \infty)$. Hence the limit $L^{+}:=\lim _{y \rightarrow \infty} \Phi_{1}(y)$ exists and is equal to zero. But, $\Phi_{1}(\bar{y}) \leq 0$ and $\Phi_{1}$ is decreasing, we have $L^{+}<0$. This contradiction implies that this case is impossible.

Case 2. $\phi(y)<1$ for all $y>\bar{y}$. This case is also impossible by the same argument as in Case 1.

We conclude that $\bar{y}=\infty$. This completes the proof.

We are ready to state and prove the main theorem of this paper as follows.

Theorem 3.6. There is no nontrivial bounded global solution $\phi$ of (1.1) such that $\left(\phi, \phi^{\prime}, \phi^{\prime \prime}\right)(\infty)=(1,0,0)$. In particular, there is no nontrivial global solution of $(1.1)$ with $\left(\phi, \phi^{\prime}, \phi^{\prime \prime}\right)( \pm \infty)=(1,0,0)$.

Proof. Suppose that $\phi$ is a nontrivial bounded global solution of (1.1) such that $\left(\phi, \phi^{\prime}, \phi^{\prime \prime}\right)(\infty)=(1,0,0)$. By Corollary 3.3, there is $y_{0} \in \mathbb{R}$ such that $\phi\left(y_{0}\right)=1$. Then $\Phi_{1}\left(y_{0}\right) \leq 0$. From Lemmas 3.4 and 3.5 it follows that there is a sequence $z_{n} \rightarrow \infty$ such that $\phi^{\prime}\left(z_{n}\right)=0$ for all $n$. Then $\Phi_{1}\left(z_{n}\right) \rightarrow 0$ as $n \rightarrow \infty$, contradicting the decreasing property of $\Phi_{1}$. The theorem follows.

\section{REFERENCES}

1. F. Bernis and L. A. Peletier, Two problems from draining flows involving third-order ordinary differential euqaitions, SIAM J. Math. Anal., 27 (1996), 515-527.

2. H.-C. Chang, Wave evolution on a falling film, Annu. Rev. Fluid Mech. 26 (1994), 103-136.

3. H.-C. Chang and E. A. Demekhin, Mechanism for drop formation on a coated vertical fibre, J. Fluid Mech. 380 (1999), 233- 255.

4. J.-S. Guo and J.-C. Tsai, The structure of solutions for a third order differential equation in boundary layer theory, Japan J. Industrial Appl. Math., 22 (2005), 311351.

5. S. Kalliadasis and H.-C. Chang, Drop formation during coating of vertical fibres, $J$. Fluid Mech., 261 (1994), 135-168. 
6. W.C. Troy, Solutions of third-order differential equations relevant to draining and coating flows, SIAM J. Math. Anal., 24 (1993), 155-171.

7. E.O. Tuck and L.W. Schwartz, A numerical and asymptotic study of some third-order ordinary differential equations relevant to draining and coating flows, SIAM Review, 32 (1990), 453-469.

\author{
Yung-Jen Lin Guo \\ Department of Mathematics, \\ National Taiwan Normal University, \\ Taipei 116, Taiwan
}

